\title{
Comparison of the performance of integrated and sequential reaction and separation units in terms of recovery of a desired product
}

\author{
Ana L. Paiva, F. Xavier Malcata* \\ Escola Superior de Biotecnologia, Universidade Católica Portuguesa, Rua Dr. Antonio Bernardino de Almeida, P-4200-072 Porto, Portugal
}

\begin{abstract}
Integration of reaction and separation has experienced fast progress in this decade because it provides a convenient way of alleviating kinetic and/or thermodynamic constraints usually present in the more traditional sequential configuration (where reaction is followed by separation), which limit the extent of reaction and also the purity of the products obtained thereby. The aim of this work was to quantitatively compare the final recovery of a desired product in an integrated processing unit and in a sequential one, in the case of a unireactant/uniproduct reaction occuring in an (ideal) liquid phase with separation via liquid/vapor equilibrium. The objective function was set as the local temperature which maximizes the recovery of product in the vapor phase leaving the integrated unit or the separator (as appropriate). It was concluded that (i) the optimum temperature looks as a local maximum for the integrated system and as a global maximum for the sequential one, (ii) it strongly depends on the equilibrium constant of the reaction in question and (iii) it assumes lower (and economically feasible) values only in the case of the integrated unit.
\end{abstract}

Keywords: Mathematical modelling; Sequential reaction and separation; Integration

\section{Introduction}

Employment of chemical reactions in obtaining a certain compound with a predefined degree of purity from a given reaction mixture classically demands subsequent downstream separation of the desired product from the unreacted feedstock and other by-products. This situation arises because thermodynamic and/or kinetic limitations raise upper limits for the extents of conversion by chemical reaction, so a compromise between yield and purity of the desired product is to be achieved. On an industrial setting, such downstream separation frequently accounts for most of the final costs associated with the manufacture of said product due to the considerable amounts of energy and overall size of apparata required, and these costs are higher when the purity specifications of the product are stricter.

In order to improve effectiveness of separation and hence reduce global costs, the possibility of continuously

\footnotetext{
*Corresponding author. Tel.: 00-351-2-5580004; fax: 00-351-25090351 .
}

removing the product(s) formed during reaction has been under scrutiny, and the latest two decades have seen a world-wide trend towards integration of reaction and separation as simultaneous steps, rather than as sequential ones (e.g. Laane et al., 1987; Tramper et al., 1992; Paiva and Malcata, 1997a,b,1999; Paiva et al., 1998,1999). Such approaches encompass (but are not limited to) integrated liquid-liquid systems (Cabral, 1991; Bart et al., 1992; Roychoudhury et al., 1995), integrated vapor-liquid systems (Davies and Jeffrey, 1973; Hills et al., 1990; Sundquist et al., 1991; De Garmo et al., 1992; Paiva and Malcata, 1994, 1996; Xu and Chuang, 1996), integrated supercritical fluid systems (Marty et al., 1992,1994), integrated solid-liquid systems (Martinek et al., 1989; Matsumura, 1991; Strathmann and Gudernatsch, 1991; van der Wielen et al., 1993,1996; van der Padt et al., 1996; Jansen, 1996; Jansen et al., 1996; Mazzotti et al., 1996) and integrated solid-gas systems (Takeuchi and Uraguchi, 1977; Parvaresh et al., 1992; Groot et al., 1992; Kemp and Macrae, 1992). A comprehensive review of application of integration in biochemical processes has been provided elsewhere (Paiva and Malcata, 1997c). 
The integrated approach, which is claimed to allow achievement of higher yields by preventing thermodynamic equilibria to be attained, was proven (Paiva et al., 1998) to decrease kinetic limitations via prevention of bulk mixing of product with (unreacted) reactant, because physical separation is achieved on the molecular level right after chemical reaction has taken place. However, it was also demonstrated that, if the overall process (i.e. reaction plus separation) rather than only a part of it (i.e. reaction) is considered, integration does not provide a true thermodynamic enhancement (Paiva and Malcata, 1997a).

Although modelling reaction and separation in integrated form is more complex than modelling said phenomena independently due to the considerably higher technical complexity of the former process, the actual decrease in the total manufacturing cost of the product owing to lower reaction times (and thus lower capital investments in smaller reactors able to effect a given conversion of reactant) coupled with separation to a higher extent (and thus lower separation costs in a posteriori less intensive separation processes) may eventually overcome the drawback associated with the aforementioned more difficult prediction. This communication aims at quantitatively addressing, on a theoretical basis, the difference in final recovery of a desired product when the performance of an integrated operation unit is compared with that of a sequential one, in the case of a reversible reaction with 1:1 stoichiometry that occurs in a liquid phase behaving as a truly ideal solution in terms of liquid/vapor equilibrium. Selection of a simple binary system rather than a ternary (possibly dilute) solution agrees with the current trend towards substitution of dilute solutions by solvent-free mixtures, thus increasing concentration of reactant to the highest possible level, and consequently obtaining a higher degree of conversion while avoiding more difficult downstream separation problems. A good example of one such reaction and system is the isomerization of 2-ethyl-1,3-dibutyl-glycerol into 3-ethyl-1,2dibutyl-glycerol, which may be catalyzed either by sodium methoxide or by lipases in water-free conditions. The importance of such reaction lies on the fact that only the outer side chains of such triglycerides (i.e. positions denoted as $s n-1$ and $s n-3$ ) are attacked by the enzymes of the human digestive tract, so only the acid residues at $s n-1$ and $s n-3$ will enter the metabolic pathways. If 2-ethyl-1,3-dibutyl-glycerol is isomerized into 3-ethyl-1,2-dibutyl-glycerol, then one less butyric acid will be released and therefore have a chance to eventually accumulate in the adipose tissues, a process often taken as unhealthy.

In this study, the objective function was defined as to maximize recovery of product in the vapor phase that leaves the integrated unit or the separator (as appropriate) in an isolated overall setting.

\section{Mathematical development}

In order to assess the differences in final recovery of a desired product from a given reactant, two alternative isenthalpic configurations will be considered as a basis for the mathematical derivations: (a) a chemical reactor integrated with a liquid/vapor separator (where vaporization occurs concomitantly with reaction); and (b) a chemical reactor (where a reaction takes place in the liquid phase) followed by a liquid/vapor separator. These two configurations are depicted in Fig. 1, and will hereafter be denoted as RS and $\mathrm{R}+\mathrm{S}$, respectively. In both situations (i) the product is assumed to be more volatile than the reactant, and (ii) the heat generated in the (exothermic) reaction step is simultaneously (or sequentially) used in full for the (endothermic) vaporization; therefore, the overall system behaves as isolated, irrespective of the actual configuration. Comparison of the performance of the two configurations will be made in terms of the temperature at which the maximum recovery of product in the vapor phase at the outlet of the RS and $\mathrm{R}+\mathrm{S}$ configurations occurs. All the mathematical development that follows will, therefore, be laid out so as to couple, for each configuration studied, all the mass balances and equilibrium equations into a single expression containing the fewest possible independent variables. In our case, only one such variable exists, and choice of temperature to play that role was based on the realization that temperature is the economic cost one has to pay in order to achieve the desired yield of product in the separation process based on vaporization.

\subsection{Integrated reaction and separation $(R S)$}

Assume that a simple, reversible chemical reaction occurring in liquid phase is stoichiometrically described by the following chemical equation:

$A \rightleftarrows B$
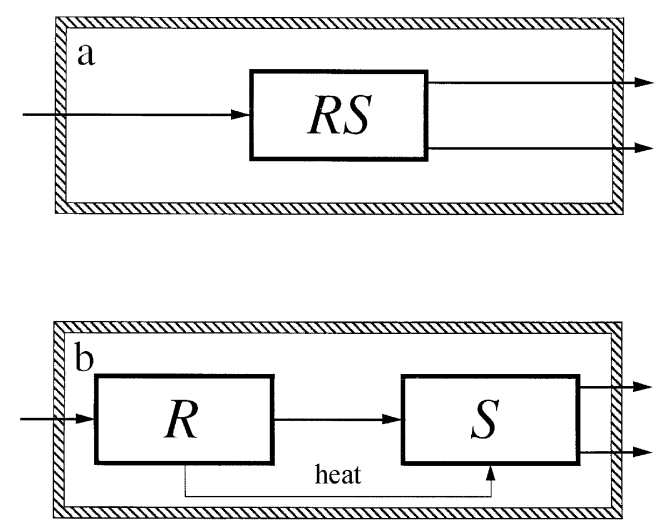

Fig. 1. Alternative configurations for the reactor and separator setup: (a) integrated (or RS) and (b) sequential (or $\mathrm{R}+\mathrm{S}$ ). Mass flows $(\longrightarrow$ ) and heat flows $(\longrightarrow$ ) are also indicated. 
where A denotes the reactant and B the product. Assuming that such binary system is an ideal solution from a thermodynamic point a view, then the chemical equilibrium should be described by

$K_{\text {eq }}\{T\}=\frac{x_{B}}{x_{A}}$,

where $x_{A}$ and $x_{B}$ denote the mole fraction, in the liquid phase, of A and B, respectively, and $T$ denotes the absolute temperature. The definition of mole fraction in a binary mixture, which implies that

$x_{A}+x_{B}=1$

allows Eq. (2) to be algebraically rearranged to yield

$x_{A}=\frac{1}{1+K_{\mathrm{eq}}\{T\}}$.

Recalling van't Hoff's law for the variation of the equilibrium constant with absolute temperature (Smith and van Ness, 1987), i.e.

$\frac{\mathrm{d} \ln \left\{K_{\mathrm{eq}}\{T\}\right\}}{\mathrm{d} T}=\frac{\Delta h^{\theta}\{T\}}{R T^{2}}$

where $\Delta h^{\theta}\{T\}$ denotes the standard enthalpy change of reaction at temperature $T$ using pure $\mathrm{A}$ and $\mathrm{B}$ at the pressure of $1 \mathrm{~atm}$ as reference (with $\Delta h^{\theta}\{T\}<0$ ), and assuming that $\Delta h^{\theta}\{T\}$ is essentially constant in the temperature range of interest (which is a consequence of assuming equal heat capacities of both reactant and product), integration of Eq. (5) leads to

$K_{\mathrm{eq}}\{T\}=K_{\mathrm{eq}}\left\{T^{\theta}\right\} \exp \left\{\frac{\Delta h^{\theta}\left\{T^{\theta}\right\}}{R}\left(\frac{1}{T^{\theta}}-\frac{1}{T}\right)\right\}$,

where $T^{\theta}$ denotes a reference (arbitrarily selected) temperature, which, for the sake of simplicity, can be taken as the (average) room temperature (i.e. $298 \mathrm{~K}$ ). The calculation of the equilibrium constant at such reference temperature may then proceed via

$K_{\mathrm{eq}}\left\{T^{\theta}\right\}=\exp \left\{-\frac{\Delta g^{\theta}\left\{T^{\theta}\right\}}{R T^{\theta}}\right\}$

where $\Delta g^{\theta}\left\{T^{\theta}\right\}$ is the standard Gibbs' free energy change of reaction at the aforementioned reference temperature. Combination of Eqs. (6) and (7) gives

$K_{\text {eq }}\{T\}=\exp \left\{\frac{\Delta s^{\theta}\left\{T^{\theta}\right\}}{R}\right\} \exp \left\{-\frac{\Delta h^{\theta}\left\{T^{\theta}\right\}}{R T}\right\}$

where advantage was taken from the fundamental property relationship, and

$\Delta g^{\theta}\left\{T^{\theta}\right\}=\Delta h^{\theta}\left\{T^{\theta}\right\}-T^{\theta} \Delta s^{\theta}\left\{T^{\theta}\right\}$, where $\Delta s^{\theta}\left\{T^{\theta}\right\}$ is the standard entropy change of reaction at temperature $T^{\theta}$. Combination of Eqs. (4) and (8) leads to

$x_{A}=\frac{1}{1+\exp \left\{\Delta s^{\theta}\left\{T^{\theta}\right\} / R\right\} \exp \left\{-\Delta h^{\theta}\left\{T^{\theta}\right\} / R T\right\}}$.

In the sequence of the reasoning explained above concerning ideal behavior, the liquid/vapor equilibrium should be described by Raoult's law, viz.

$x_{A} P_{A}^{\sigma}\{T\}=y_{A} P$,

and

$x_{B} P_{B}^{\sigma}\{T\}=y_{B} P$,

where $y_{A}$ and $y_{B}$ denote the mole fraction, in the vapor phase, of $\mathrm{A}$ and $\mathrm{B}$, respectively, $P$ denotes absolute pressure, and $P_{A}^{\sigma}\{T\}$ and $P_{B}^{\sigma}\{T\}$ denote the saturation pressure of $\mathrm{A}$ and $\mathrm{B}$, respectively, at the given temperature. Recalling again Eq. (3) coupled with its vaporphase counterpart, viz.

$y_{A}+y_{B}=1$

and combining Eqs. (11) and (12), one obtains

$P=P_{B}^{\sigma}\{T\}+\left(P_{A}^{\sigma}\{T\}-P_{B}^{\sigma}\{T\}\right) x_{A}$

Using Eq. (14) allows Eq. (11) to read

$y_{A}=\frac{P_{A}^{\sigma}\{T\} x_{A}}{P_{B}^{\sigma}\{T\}+\left(P_{A}^{\sigma}\{T\}-P_{B}^{\sigma}\{T\}\right) x_{A}}$.

If reaction equilibrium conditions prevail in the liquid phase, which is simultaneously in equilibrium with the vapor phase (as implied by configuration RS), then Eqs. (4) and (15) may be combined to give

$y_{A}=\frac{P_{A}^{\sigma}\{T\}}{P_{A}^{\sigma}\{T\}+P_{B}^{\sigma}\{T\} K_{\mathrm{eq}}\{T\}}$.

Hence, the composition in $\mathrm{A}$ of both the liquid and the vapor phase, as depicted in Eqs. (4) and (16), respectively, depends (solely) on the actual temperature considered.

The variation of the vapor pressure of a pure compound $i$ with temperature can be obtained in its simplest (accepted) form as

$P_{i}^{\sigma}\{T\}=\exp \left\{\alpha_{i}-\frac{\beta_{i}}{T}\right\}, \quad i=\mathrm{A}, \mathrm{B}$,

where $\alpha_{i}$ and $\beta_{i}$ are empiric parameters associated with compound $i$ (with $P_{B}^{\sigma}\{T\}>P_{A}^{\sigma}\{T\}$, as required by the higher volatility of B). Combination of Eqs. (8), (16) and 
(17) finally yields

$$
y_{A}=\frac{1}{1+\exp \left\{\alpha_{B}-\alpha_{A}+\Delta s^{\theta}\left\{T^{\theta}\right\} / R\right\} \exp \left\{-\left(\beta_{B}-\beta_{A}+\Delta h^{\theta}\left\{T^{\theta}\right\} / R\right) / T\right\}} .
$$

The equality of Gibbs free energy for two phases in equilibrium permits derivation of Clapeyron equation, viz.

$$
\Delta h_{i}^{\operatorname{vap}}\{T\}=T \Delta v_{i}^{\operatorname{vap}}\left(\frac{\mathrm{d} P^{\sigma}}{\mathrm{d} T}\right),
$$

where $\Delta h_{i}^{\text {vap }}$ and $\Delta v_{i}^{\text {vap }}$ are the enthalpy change and volume change of vaporization, respectively, of compound $i$. Recalling the initial assumption that the vapor phase behaves as an ideal gas (which implies that each component is well described by the ideal gas equation of state) and coupling it with the reasonable assumption that the molar volume of the liquid phase is negligible when compared with that of the vapor phase, one gets

$$
\Delta h_{i}^{\mathrm{vap}}\{T\}=-R \frac{\mathrm{d} \ln \left\{P_{i}^{\sigma}\{T\}\right\}}{\mathrm{d}\{1 / T\}}
$$

which, combined with Eq. (17), becomes

$$
\Delta h_{i}^{\mathrm{vap}}=R \beta_{i}
$$

also known as Clausius-Clapeyron equation (Smith and van Ness, 1987).

The overall molar balance to the RS system can be written as

$$
N_{\text {tot }}=N_{L}+N_{V},
$$

where $N_{\text {tot }}$ is the total number of moles in the system, and $N_{L}$ and $N_{V}$ are the total numbers of moles (of both A and B) in the liquid and vapor phases, respectively. Assuming that the inlet to the integrated system is pure A at temperature $T^{\theta}$, then the total enthalpy change due to reaction, $\Delta H^{r x n}$, is given by

$$
\Delta H^{r x n}\{T\}=N_{\text {tot }} c_{A}^{\theta}\left(T-T^{\theta}\right)+\left(N_{L} x_{B}+N_{V} y_{B}\right) \Delta h^{\theta}\left\{T^{\theta}\right\},
$$

where $c_{A}^{\theta}$ (which is assumed to be independent of temperature) is the (molar) specific heat capacity of reactant A (which, for that matter, is equal to that of $\mathrm{B}$ ) at the aforementioned temperature, and where the simplifying assumption that the enthalpy change of reaction is virtually independent of temperature was again considered. Combination of Eqs. (3), (10), (13), (18), (22) and (23) finally leads to the following expression for the total enthalpy change between initial loading and final attainment of equilibrium

$$
\begin{aligned}
\frac{\Delta H^{r x n}}{N_{\text {tot }}}= & c_{A}^{\theta}\left(T-T^{\theta}\right)+\frac{(1-\zeta V) \exp \left\{\Delta s^{\theta}\left\{T^{\theta}\right\} / R\right\} \exp \left\{-\Delta h^{\theta}\left\{T^{\theta}\right\} / R T\right\} \Delta h^{\theta}\left\{T^{\theta}\right\}}{1+\exp \left\{\Delta s^{\theta}\left\{T^{\theta}\right\} / R\right\} \exp \left\{-\Delta h^{\theta}\left\{T^{\theta}\right\} / R T\right\}} \\
& +\frac{\zeta V \exp \left\{\alpha_{B}-\alpha_{A}+\Delta s^{\theta}\left\{T^{\theta}\right\} / R\right\} \exp \left\{-\left(\beta_{B}-\beta_{A}+\Delta h^{\theta}\left\{T^{\theta}\right\} / R\right) / T\right\} \Delta h^{\theta}\left\{T^{\theta}\right\}}{1+\exp \left\{\alpha_{B}-\alpha_{A}+\Delta s^{\theta}\left\{T^{\theta}\right\} / R\right\} \exp \left\{-\left(\beta_{B}-\beta_{A}+\Delta h^{\theta}\left\{T^{\theta}\right\} / R\right) / T\right\}},
\end{aligned}
$$

where the fractional degree of vaporization, $\zeta_{V}$, is given by

$$
\zeta_{V}=\frac{N_{V}}{N_{\text {tot }}}
$$

and where again the assumption that the molar heat capacity at the reference conditions is essentially constant within the temperature range of interest was taken.

On the other hand, the total enthalpy change due to vaporization, $\Delta H^{\text {vap }}$, is given by

$$
\Delta H^{\text {vap }}\{T\}=N_{V}\left(y_{A} \Delta h_{A}^{\mathrm{vap}}+y_{B} \Delta h_{B}^{\mathrm{vap}}\right),
$$

where advantage was taken from the nil enthalpy change of mixing that is a characteristic of ideal solutions. Upon combination of Eqs. (13) and (21), Eq. (26) becomes

$$
\Delta H^{\text {vap }}\{T\}=N_{V} R\left(\beta_{B}+\left(\beta_{A}-\beta_{B}\right) y_{A}\right)
$$

which, in view of Eq. (18), can be algebraically rearranged to read

$$
\frac{\Delta H^{\mathrm{vap}}\{T\}}{N_{\mathrm{tot}}}=\zeta_{V} R\left(\frac{\beta_{A}+\beta_{B} \exp \left\{\alpha_{B}-\alpha_{A}+\Delta s^{\theta}\left\{T^{\theta}\right\} / R\right\} \exp \left\{-\left(\beta_{B}-\beta_{A}+\Delta h^{\theta}\left\{T^{\theta}\right\} / R\right) / T\right\}}{1+\exp \left\{\alpha_{\beta}-\alpha_{A}+\Delta s^{\theta}\left\{T^{\theta}\right\} / R\right\} \exp \left\{-\left(\beta_{B}-\beta_{A}+\Delta h^{\theta}\left\{T^{\theta}\right\} / R\right) / T\right\}}\right)
$$


The overall enthalpy balance will, as a result of imposing the isenthalpic condition, take the following form:

$$
\Delta H^{r x n}\{T\}+\Delta H^{\operatorname{vap}}\{T\}=0
$$

Hence, combination of Eqs. (24), (28) and (29) leads to

$$
\begin{aligned}
& c_{A}^{\theta}\left(T-T^{\theta}\right)+\frac{(1-\zeta V) \exp \left\{\Delta s^{\theta}\left\{T^{\theta}\right\} / R\right\} \exp \left\{-\Delta h^{\theta}\left\{T^{\theta}\right\} / R T\right\} \Delta h^{\theta}\left\{T^{\theta}\right\}}{1+\exp \left\{\Delta s^{\theta}\left\{T^{\theta}\right\} / R\right\} \exp \left\{-\Delta h^{\theta}\left\{T^{\theta}\right\} / R T\right\}} \\
& +\frac{\zeta V\left(R \beta_{A}+\left(\Delta h^{\theta}\left\{T^{\theta}\right\}+R \beta_{B}\right) \exp \left\{\alpha_{B}-\alpha_{A}+\Delta s^{\theta}\left\{T^{\theta}\right\} / R\right\} \exp \left\{-\left(\beta_{B}-\beta_{A}+\Delta h^{\theta}\left\{T^{\theta}\right\} / R\right) / T\right\}\right.}{1+\exp \left\{\alpha_{B}-\alpha_{A}+\Delta s^{\theta}\left\{T^{\theta}\right\} / R\right\} \exp \left\{-\left(\beta_{B}-\beta_{A}+\Delta h^{\theta}\left\{T^{\theta}\right\} / R\right) / T\right\}}=0
\end{aligned}
$$

and solution of Eq. (30) with respect to $\zeta_{V}$ finally gives

$$
\zeta_{V}=\frac{c_{A}^{*}\left(T^{*}-1\right)+\frac{\exp \left\{\Delta s^{*}\right\} \exp \left\{-\Delta h^{*} / T^{*}\right\}}{1+\exp \left\{\Delta s^{*}\right\} \exp \left\{-\Delta h^{*} / T^{*}\right\}}}{\frac{\exp \left\{\Delta s^{*}\right\} \exp \left\{-\Delta h^{*} / T^{*}\right\}}{1+\exp \left\{\Delta s^{*}\right\} \exp \left\{-\Delta h^{*} / T^{*}\right\}}-\frac{\left(\beta_{A}^{*}+\left(\Delta h^{*}+\beta_{B}^{*}\right) \exp \left\{\alpha_{B}-\alpha_{A}+\Delta s^{*}\right\} \exp \left\{-\left(\beta_{B}^{*}-\beta_{A}^{*}+\Delta h^{*}\right) / T^{*}\right\}\right)}{\Delta h^{*}\left(1+\exp \left\{\alpha_{B}-\alpha_{A}+\Delta s^{*}\right\} \exp \left\{-\left(\beta_{B}^{*}-\beta_{A}^{*}+\Delta h^{*}\right) / T^{*}\right\}\right)}}
$$

where the parameters used are defined as

$$
\begin{aligned}
& c_{A}^{*}=\frac{c_{A}^{\theta} T^{\theta}}{\Delta h^{\theta}} \\
& \Delta s^{*}=\frac{\Delta s^{\theta}}{R} \\
& \Delta h^{*}=\frac{\Delta h^{\theta}}{R T^{\theta}}, \\
& \beta_{i}^{*}=\frac{\beta_{i}}{T^{\theta}}, \quad i=\mathrm{A}, \mathrm{B}
\end{aligned}
$$

and

$$
T^{*}=\frac{T}{T^{\theta}}
$$

Since there is one degree of freedom left (i.e. the temperature, $T^{*}$ ), then this can be used as predictor in an optimization procedure after having selected as objective function the total amount of B recovered in the gaseous outlet, viz.

$$
\frac{\mathrm{d}\left(\zeta_{V} y_{B}\right)}{\mathrm{d} T^{*}}=0
$$

which may be applied after combination of Eqs. (13), (18) and (31), i.e. to

$$
\zeta_{V} y_{B}=\frac{\left(c_{A}^{*}\left(T^{*}-1\right)+\frac{\exp \left\{\Delta s^{*}\right\} \exp \left\{-\Delta h^{*} / T^{*}\right\}}{1+\exp \left\{\Delta s^{*}\right\} \exp \left\{-\Delta h^{*} / T^{*}\right\}}\right)\left(\frac{\exp \left\{\alpha_{B}-\alpha_{A}+\Delta s^{*}\right\} \exp \left\{-\left(\beta_{B}^{*}-\beta_{A}^{*}+\Delta h^{*}\right) / T^{*}\right\}}{\left(1+\exp \left\{\alpha_{B}-\alpha_{A}+\Delta s^{*}\right\} \exp \left\{-\left(\beta_{B}^{*}-\beta_{A}^{*}+\Delta h^{*}\right) / T^{*}\right\}\right.}\right)}{\frac{\exp \left\{\Delta s^{*}\right\} \exp \left\{-\Delta h^{*} / T^{*}\right\}}{1+\exp \left\{\Delta s^{*}\right\} \exp \left\{-\Delta h^{*} / T^{*}\right\}}-\frac{\left(\beta_{A}^{*}+\left(\Delta h^{*}+\beta_{B}^{*}\right) \exp \left\{\alpha_{B}-\alpha_{A}+\Delta s^{*}\right\} \exp \left\{-\left(\beta_{B}^{*}-\beta_{A}^{*}+\Delta h^{*}\right) / T^{*}\right\}\right)}{\Delta h^{*}\left(1+\exp \left\{\alpha_{B}-\alpha_{A}+\Delta s^{*}\right\} \exp \left\{-\left(\beta_{B}^{*}-\beta_{A}^{*}+\Delta h^{*}\right) / T^{*}\right\}\right)}} .
$$

Plots of $\zeta_{V} y_{B}$ as a function of $T^{*}$ are available in Fig. 2 for different values of $K_{\text {eq }}$, where $K_{\text {eq }}$ was calculated by means of the dimensionless counterpart of Eq. (8) through manipulation of $\Delta H$. Parameters $c_{A}, \alpha_{A}, \alpha_{B}, \beta_{A}, \beta_{B}, \Delta h$ and $\Delta s$ used in the calculations were given values typically found in the literature (e.g. Perry, 1984) so as to guarantee both the applicability of the derivation and the validity of the assumptions made, i.e. $P_{B}^{\sigma}>P_{A}^{\sigma}$ (product B more volatile than reactant $\mathrm{A}$ ) and $\Delta H<0$ (exothermic reaction). 


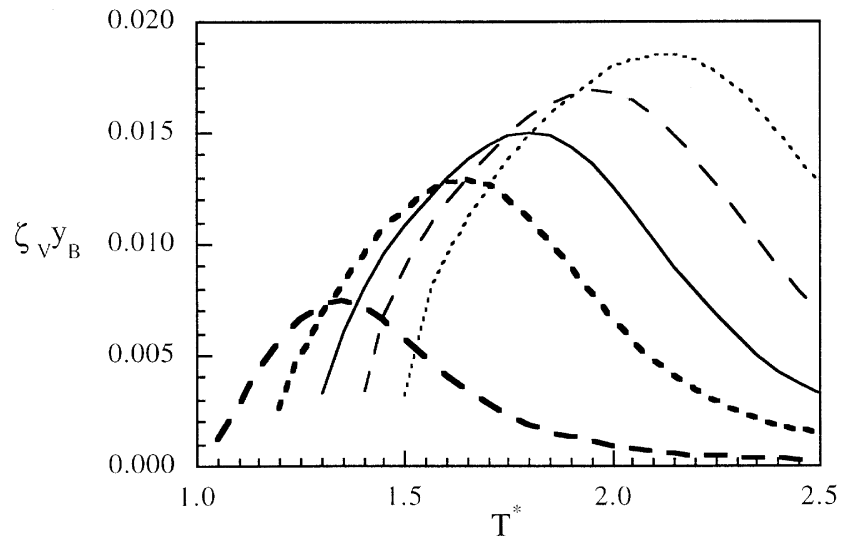

Fig. 2. Plot of the fractional recovery, $\zeta_{V} y_{B}$, vs. the absolute temperature, $T^{*}$, for the RS configuration, for $c_{A}=5 \mathrm{~J} /(\mathrm{mol} \mathrm{K})$,

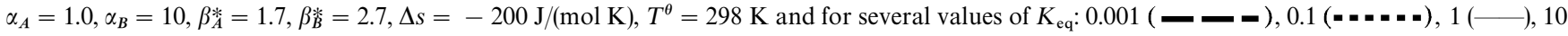
$(--\longrightarrow)$ and $100(-\cdots-\cdot)$.

\subsection{Sequential reaction and separation}

Consider now the $\mathrm{R}+\mathrm{S}$ configuration where the reaction depicted as Eq. (1) (and assumed to be exothermic, as before) is carried out in a reactor followed by a separator. In this case, the total enthalpy change due to reaction, $\Delta H^{r x n}$, is given by

$$
\Delta H^{r x n}\{T\}=N_{\text {tot }} z_{A} \Delta h^{\theta}\left\{T^{\theta}\right\},
$$

where the inlet and outlet streams are at the reference temperature $T^{\theta}$. Here $z_{A}$ denotes the mole fraction of $\mathrm{A}$ in the (liquid) outlet of the reactor, so it is given by

$$
z_{A}=\frac{1}{1+K_{\mathrm{eq}}\left\{T^{\theta}\right\}}
$$

as an analog of Eq. (4), since the conversion of the reactant into product is limited by the equilibrium conditions. Substitution of Eq. (40) in Eq. (39) then gives

$$
\Delta H^{r x n}\left\{T^{\theta}\right\}=N_{\text {tot }} \frac{\Delta h^{\theta}\left\{T^{\theta}\right\}}{1+K_{\text {eq }}\left\{T^{\theta}\right\}},
$$

where $\Delta H^{r x n}$ is explicitly taken as a constant because it depends only on $T$ which, in view of the isothermal behavior assumed in the reactor, is equal to $T^{\theta}$. The heat generated in the reactor, as given by Eq. (41), is used in the separator (i) to increase the temperature of the inlet stream from $T^{\theta}$ to its outlet temperature, $T$, (ii) to evaporate part of reactant $\mathrm{A}$, and (iii) to evaporate part of product $\mathrm{B}$. The heat necessary to accomplish such tasks, $\Delta H^{\text {sep }}$, is thus given by

$$
\Delta H^{\mathrm{sep}}=N_{\mathrm{tot}} \theta_{A}^{\theta}\left(T-T^{\theta}\right)+N_{V} \Delta h_{A}^{\mathrm{vap}}\left(1-y_{B}\right)+N_{V} \Delta h_{B}^{\mathrm{vap}} y_{B},
$$

where advantage was taken from the assumption made before that $c_{A}^{\theta}=c_{B}^{\theta}$.

Recalling Eq. (21), (42) now becomes

$$
\Delta H^{\text {sep }}=N_{\text {tot }} c_{A}^{\theta}\left(T-T^{\theta}\right)+R N_{V}\left(\beta_{A}+\left(\beta_{B}-\beta_{A}\right) y_{B}\right) .
$$

Recalling the assumption of overall adiabatic processing, then

$$
\Delta H^{r x n}+\Delta H^{\mathrm{sep}}=0
$$

Substitution of Eqs. (25), (39) and (43) in Eq. (44) leads to

$$
\zeta_{V}=\frac{c_{A}^{\theta}\left(T^{\theta}-T\right)-\Delta h^{\theta} z_{A}}{R\left\{\beta_{A}-\beta_{A} y_{B}+\beta_{B} y_{B}\right\}}
$$

which, upon algebraic manipulation and combination with Eqs. (32)-(36), gives

$$
\zeta_{V}=\frac{\Delta h^{*}\left\{c_{A}^{*}\left(1-T^{*}\right)-z_{A}\right\}}{\beta_{A}^{*}+\left(\beta_{B}^{*}-\beta_{A}^{*}\right) y_{B}},
$$


where substitution of $z_{A}$ by Eq. (40) gives

$$
\zeta_{V}=\frac{\Delta h^{*}\left\{c_{A}^{*}\left(1+K_{\mathrm{eq}}\left\{T^{\theta}\right\}\right)\left(1-T^{*}\right)-1\right\}}{\left(1+K_{\mathrm{eq}}\left\{T^{\theta}\right\}\right)\left(\beta_{A}^{*}+\left(\beta_{B}^{*}-\beta_{A}^{*}\right) y_{B}\right)},
$$

and where $K_{\text {eq }}$ is calculated from the dimensionless counterpart of Eq. (8), viz.

$$
K_{\text {eq }}\left\{T^{\theta}\right\}=\exp \left\{\Delta s^{*}\right\} \exp \left\{-\Delta h^{*} T^{*}\right\}
$$

The molar balance to component $\mathrm{A}$ in the separator can be written as

$$
N_{\text {tot }} z_{A}=N_{L} x_{A}+N_{V} y_{A},
$$

where $N_{L}$ and $N_{V}$ are the total number of moles (of both A and B) in the liquid phase and in the vapor phase, respectively, at any time and $N_{\text {tot }}$ is given by Eq. (22).

Recalling Eq. (25) and combining it with Eqs. (22) and (49), one may write

$$
\zeta_{V}=\frac{N_{V}}{N_{\text {tot }}}=\frac{z_{A}-x_{A}}{y_{A}-x_{A}}
$$

which, upon solution with respect to $z_{A}$, yields

$$
z_{A}=x_{A}+\zeta_{V}\left(y_{A}-x_{A}\right)
$$

where $x_{A}$, the molar fraction of $\mathrm{A}$ in the liquid stream, can be expressed as a function of $y_{A}, P_{A}^{\sigma}$ and $P_{B}^{\sigma}$ through algebraic manipulation of Eq. (15), using Eqs. (17), (35) and (36), viz.

$$
x_{A}=\frac{y_{A} \exp \left\{\alpha_{B}-\beta_{B}^{*} / T^{*}\right\}}{\exp \left\{\alpha_{A}-\beta_{A}^{*} / T^{*}\right\}+y_{A}\left(\exp \left\{\alpha_{B}-\beta_{B}^{*} / T^{*}\right\}-\exp \left\{\alpha_{A}-\beta_{A}^{*} / T^{*}\right\}\right)} .
$$

Substitution of Eq. (52) in Eq. (51) then gives

$$
z_{A}=\left(1-y_{B}\right)\left\{\frac{\exp \left\{\alpha_{B}-\beta_{B}^{*} / T^{*}\right\}+\zeta_{V} y_{B}\left(\exp \left\{\alpha_{A}-\beta_{A}^{*} / T^{*}\right\}-\exp \left\{\alpha_{B}-\beta_{B}^{*} / T^{*}\right\}\right)}{\exp \left\{\alpha_{B}-\beta_{B}^{*} / T^{*}\right\}+y_{B}\left(\exp \left\{\alpha_{A}-\beta_{A}^{*} / T^{*}\right\}-\exp \left\{\alpha_{B}-\beta_{B}^{*} / T^{*}\right\}\right)}\right\}
$$

From combination of Eqs. (40) and (53), one obtains, after simplification, a quadratic equation in $y_{B}$, viz.

$$
\begin{aligned}
\zeta_{V} & \left\{\exp \left(\alpha_{A}-\beta_{A}^{*} / T^{*}\right)-\exp \left(\alpha_{B}-\beta_{B}^{*} / T^{*}\right)\right\}\left(1+K_{\mathrm{eq}}\left\{T^{\theta}\right\}\right) y_{B}^{2} \\
& +\left\{K_{\mathrm{eq}} \exp \left(\alpha_{B}-\beta_{B}^{*} / T^{*}\right)+\exp \left(\alpha_{A}-\beta_{A}^{*} / T^{*}\right)\right. \\
& \left.-\zeta_{V}\left(\exp \left(\alpha_{A}-\beta_{A}^{*} / T^{*}\right)-\exp \left(\alpha_{B}-\beta_{B}^{*} / T^{*}\right)\right)\left(1+K_{\mathrm{eq}}\right)\right\} y_{B} \\
& -K_{\mathrm{eq}}\left\{T^{\theta}\right\} \exp \left(\alpha_{B}-\beta_{B}^{*} / T^{*}\right)=0
\end{aligned}
$$

Solution of this expression with respect to $y_{B}$ then gives

$$
\begin{aligned}
&- K_{\mathrm{eq}}\left\{T^{\theta}\right\} \exp \left(\alpha_{B}-\beta_{B}^{*} / T^{*}\right)-\exp \left(\alpha_{A}-\beta_{A}^{*} / T^{*}\right)+\zeta_{V}\left(1+K_{\mathrm{eq}}\left\{T^{\theta}\right\}\right)\left\{\exp \left(\alpha_{A}-\beta_{A}^{*} / T^{*}\right)-\exp \left(\alpha_{B}-\beta_{B}^{*} / T^{*}\right)\right\} \\
&+\sqrt{\begin{array}{c}
\left\{K_{\mathrm{eq}}\left\{T^{\theta}\right\} \exp \left(\alpha_{B}-\beta_{B}^{*} / T^{*}\right)+\exp \left(\alpha_{A}-\beta_{A}^{*} / T^{*}\right)-\zeta_{V}\left(1+K_{\mathrm{eq}}\left\{T^{\theta}\right\}\right)\left[\exp \left(\alpha_{A}-\beta_{A}^{*} / T^{*}\right)-\exp \left(\alpha_{B}-\beta_{B}^{*} / T^{*}\right)\right]\right\}^{2} \\
+4 \exp \left(\alpha_{B}-\beta_{B}^{*} / T^{*}\right) K_{\mathrm{eq}}\left\{T^{\theta}\right\} \zeta_{V}\left[\exp \left(\alpha_{A}-\beta_{A}^{*} / T^{*}\right)-\exp \left(\alpha_{B}-\beta_{B}^{*} / T^{*}\right)\right]\left(1+K_{\mathrm{eq}}\left\{T^{\theta}\right\}\right)
\end{array}} \\
& y_{B}=\frac{\sqrt{\zeta_{V}\left(1+K_{\mathrm{eq}}\left\{T^{\theta}\right\}\right)\left\{\exp \left(\alpha_{A}-\beta_{A}^{*} / T^{*}\right)-\exp \left(\alpha_{B}-\beta_{B}^{*} / T^{*}\right)\right\}}}{}
\end{aligned}
$$

where only the plus sign was considered because the minus sign yielded mole fractions of $\mathrm{B}$, in the vapor phase, above unity. An explicit expression for $\zeta_{V}$ can now be obtained by substitution of Eq. (55) in Eq. (47). Plots of $\zeta_{V} y_{B}$ as a function of $T^{*}$ are available in Fig. 3 for different values of $K_{\text {eq }}$, where $K_{\text {eq }}$ was calculated by means of Eq. (48) through manipulation of $\Delta H$. Parameters $c_{A}, \alpha_{A}, \alpha_{B}, \beta_{A}, \beta_{B}, \Delta h$ and $\Delta s$ used in the calculations were given values typically found 


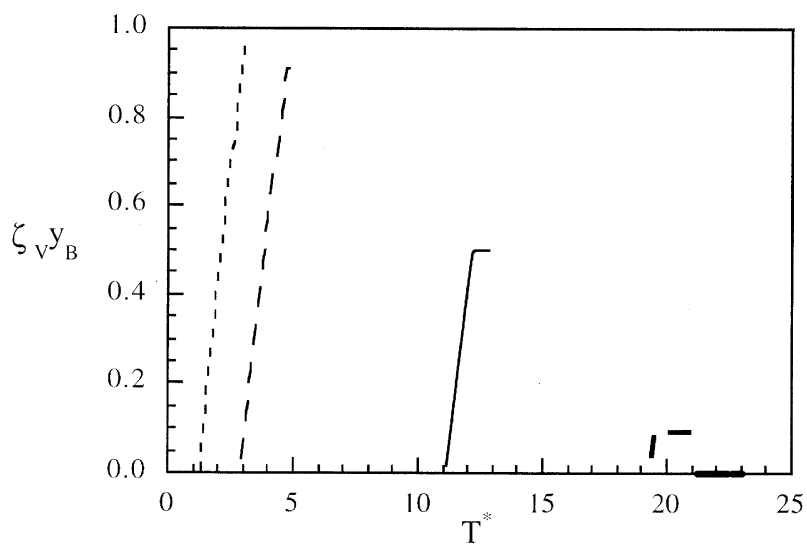

Fig. 3. Plot of the fractional recovery, $\zeta_{V} y_{B}$, vs. the absolute temperature, $T^{*}$, for the $\mathrm{R}+\mathrm{S}$ configuration, for $\mathrm{c}_{\mathrm{A}}=5 \mathrm{~J} /(\mathrm{mol} \mathrm{K})$, $\alpha_{A}=1.0, \alpha_{B}=10, \beta_{A}^{*}=1.7, \beta_{B}^{*}=2.7, \Delta s=-200 \mathrm{~J} /(\mathrm{mol} \mathrm{K})$, $T^{\theta}=298 \mathrm{~K}$ and for several values of $K_{\text {eq }}: 0.001$ (- - $), 0.1$

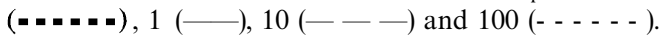

in the literature (e.g. Perry, 1984) so as to guarantee both the applicability of the derivation and the validity of the assumptions made, i.e. $P_{B}^{\sigma}>P_{A}^{\sigma}$ (product B more volatile than reactant $\mathrm{A})$ and $\Delta H<0$ (exothermic reaction).

\section{Discussion}

In this work, the two alternative configurations depicted in Fig. 1 were considered: (a) the integrated RS configuration, where reaction and separation (via vaporization) occur simultaneously; and (b) the $\mathrm{R}+\mathrm{S}$ configuration, where separation (also via vaporization) occurs after reaction has taken place. In both situations the overall system was assumed to be perfectly isolated, which means that all the heat generated in the (exothermic) reaction step was used in the vaporization of both A and B in the separation step. For the sake of physicochemical feasibility, enthalpy change of reaction was assumed to take negative values. Also, and in order for the separation via vaporization be effective, the product (B) was considered to be more volatile than the reactant (A), i.e. parameters $\alpha$ and $\beta$ used in Eq. (17) for each compound were chosen so that the vapor pressure of the product is higher than the vapor pressure of the reactant at every temperature of interest.

Both configurations were assumed to depart from the same initial load of reactant at the same temperature and, as they do not exchange energy or mass in any form with the surroundings, the final states would be expected to coincide from a thermodynamic point of view, with consequent lack of relative advantage of the integration approach with respect to the unit operation approach, as emphasized elsewhere by Paiva and Malcata (1997a). However, it should be noted that while the RS configura- tion is operated reversibly (so a true equilibrium is allowed to be reached for the chemical reaction and phase change processes), the same does not hold for the $\mathrm{R}+\mathrm{S}$ configuration because no vapor is allowed to form in the reactor unit where only reaction equilibrium is assumed to be attained; furthermore, even if a tiny bubble would form to ensure liquid/vapor equilibrium, the reaction would not be allowed to proceed in the separator unit (owing to absence of catalyst or excessively short average residence time), which then would prevent a true thermodynamic equilibrium to be reached in the $\mathrm{R}+\mathrm{S}$ system.

Comparison of performance of the two configurations was then made in terms of the temperature at which the maximum recovery of product in the vapor phase $\left(\zeta_{V} y_{B}\right)$ at the outlet of the RS and $\mathrm{R}+\mathrm{S}$ configurations occurs, for five orders of magnitude of the equilibrium constant. In fact, the problem tackled leads, as shown, to one degree of freedom (i.e. temperature), a realization that is in agreement with Gibbs phase rule and Duhem theorem (Smith and van Ness, 1987). As a matter of fact, in both configurations considered, there are two compounds, two phases and one chemical reaction, which leads to one intensive degree of freedom; on the other hand, given the initial amounts of each compound, there are (invariably) two total degrees of freedom, which in our case (and due to the rationale above) implies that one of them must be extensive. Since an extra enthalpy balance was considered, then such extensive degree of freedom (accounted in our case by, say, the total number of moles in the vapor phase $N_{V}$ ) could be removed and only one intensive degree of freedom (i.e. temperature) was eventually left. In practical terms, temperature may alternatively be controlled via control of overall pressure in the system.

When the integrated RS configuration is considered, one concludes from inspection of Fig. 2 that there is always a temperature value for which a local maximum degree of recovery of product B in the vapor phase is attained. Recalling that the actual values of temperature were previously normalized by $298 \mathrm{~K}$ in order to produce the axis label in the plot, one can see that the maximum recovery of product (which ranges from 0.007 to 0.019 when the equilibrium constant ranges from 0.001 to 100) occurs always at economically feasible temperatures (ranging from ca. 387 to $626 \mathrm{~K}$, respectively) and depends strongly on the extent of reaction: for smaller values of $K_{\text {eq }},\left(\zeta_{V} y_{B}\right)_{\text {opt }}$ is lower and $T_{\text {opt }}^{*}$ is also lower. The reason why increases in $K_{\text {eq }}$ in the integrated configuration promote a positive shift of such optimum is a consequence of less tight thermodynamic constraints upon the chemical reaction, which allow temperature (which leads to unfavourable effects due to the exothermic nature of the reaction) take higher values.

From inspection of Fig. 3 one can see that, unlike what happens with the RS configuration, a local maximum of recovery of product in the vapor stream never occurs. In 


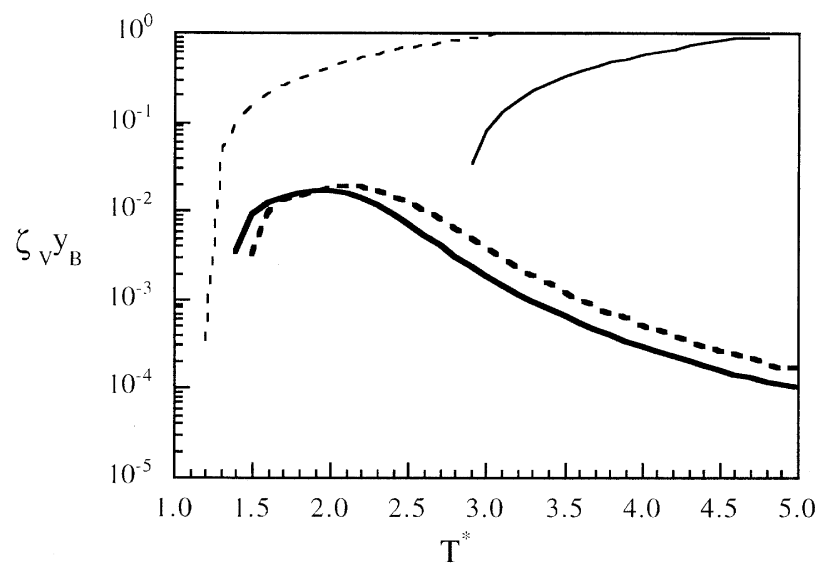

Fig. 4. Plot of the fractional recovery, $\zeta_{V} y_{B}$, vs. the absolute temperature, $T^{*}$, for the RS (thick lines) and $\mathrm{R}+\mathrm{S}$ (thin lines) configurations, for $c_{A}=5 \mathrm{~J} /(\mathrm{mol} \mathrm{K}), \alpha_{A}=1.0, \alpha_{B}=10, \beta_{A}^{*}=1.7, \beta_{B}^{*}=2.7, \Delta s=-200 \mathrm{~J} /$ $(\mathrm{mol} \mathrm{K}), T^{\theta}=298 \mathrm{~K}$ and for two values of $K_{\mathrm{eq}}: 10(-)$ and 100 $(-\cdots-\cdot-)$

this situation, for a similar range of values of the equilibrium constant, the curves obtained are in fact limited to a very narrow range of temperature values due to constraints (i.e. maximum values borne by the compounds before thermal cracking or by the container material before melting). Inspection of Fig. 3 also indicates that, in spite of the higher yields of product obtained in the vapor stream when compared to the ones obtained under the same conditions in the RS configuration, only for reactions characterized by $K_{\text {eq }}$ above, say 10 does the $\mathrm{R}+\mathrm{S}$ configuration provide economically feasible results (measured in terms of temperature). Such trend may be explained by the fact that increasing $K_{\text {eq }}$ leads to increases, not only of the amount of B produced, but also of the amount of heat available for vaporization (because the reaction is exothermic) and hence of the amount of $B$ recovered as vapor.

A comparison of the objective function $\zeta_{V} y_{B}$ as a function of $T^{*}$ for both configurations $\mathrm{RS}$ and $\mathrm{R}+\mathrm{S}$ for values of $K_{\text {eq }}$ of 10 and 100 can be seen in Fig. 4 where, for the sake of easier perception, a semilog scale was used. From inspection of this plot one can see that, although for the RS configuration the temperatures at which the maximum in the objective function is achieved are lower, such maximum is much below that of the values that can be attained using the $\mathrm{R}+\mathrm{S}$ configuration at the same temperatures. Moreover, slightly higher temperatures can lead to almost full recoveries, therefore making the use of the $\mathrm{R}+\mathrm{S}$ configuration very interesting (the more interesting the higher the $K_{\text {eq }}$ of the reaction under study, since temperatures are shifted towards lower values).

\section{Conclusions}

The previous considerations have indicated that: (i) the optimum product recovery occurs at lower (and phys- ically realizable) temperatures in the case of integrated reaction and separation; (ii) the equilibrium constant plays an important role in discriminating between the RS and the $\mathrm{R}+\mathrm{S}$ configurations; (iii) an increase in $K_{\text {eq }}$ promotes a shift of the optimum temperature to higher values in the case of the integrated approach; (iv) an increase in $K_{\text {eq }}$ promotes a shift of the optimum temperature to lower values in the case of the sequential approach; and (v) the sequential configuration provides better results (in both economics of the process and recovery of product) for high values of $K_{\text {eq }}$ (say, greater than 10).

If the likely more common situation of a dilute reaction system were considered, e.g. a reversible reaction with two reactants (A and D) and one product (B), then the results obtained would strongly depend on the relative volatilities of the components involved. If one considers that reactant $\mathrm{D}$ is much less volatile than $\mathrm{A}$, then the difference between the $\mathrm{RS}$ and the $\mathrm{R}+\mathrm{S}$ configurations (assuming that once again one wanted to maximize recovery of product $\mathrm{B}$ ) would be less apparent owing to the damping volatility effect of the other reactant $(\mathrm{A})$.

\section{Notation}

A substrate, Dimensionless

B product, Dimensionless

$c_{i}$ specific heat capacity of component $i$, $\mathrm{J} \mathrm{mol}^{-1} \mathrm{~K}^{-1}$

$K_{\text {eq }} \quad$ equilibrium constant, Dimensionless

$N \quad$ number of moles, mol

$P \quad$ absolute pressure, $\mathrm{Pa}$

$P_{i}^{\sigma} \quad$ saturation pressure of pure component $i, \mathrm{~Pa}$

$R \quad$ ideal gas constant, $\mathrm{J} \mathrm{mol}^{-1} \mathrm{~K}^{-1}$

$\mathrm{R} \quad$ reactor, Dimensionless

RS integrated reaction and separation, dimensionless

$\mathrm{R}+\mathrm{S}$ sequential reaction and separation, dimensionless

S separator, Dimensionless

$T$ temperature, $\mathrm{K}$

$x_{i} \quad$ molar fraction of component $i$ in the liquid phase, Dimensionless

$y_{i} \quad$ molar fraction of component $i$ in the vapor phase, Dimensionless

$z_{i} \quad$ molar fraction of component $i$ in the inlet liquid phases to the separator, Dimensionless

\section{Greek letters}

$\alpha_{i} \quad$ empiric parameter associated with component $i$, Dimensionless

$\beta_{i} \quad$ empiric parameter associated with component $i, \mathrm{~K}$

$\Delta g \quad$ molar Gibb's free energy of reaction, $\mathrm{J} \mathrm{mol}^{-1}$

$\Delta H \quad$ enthalpy change, $\mathrm{J}$

$\Delta h \quad$ specific enthalpy change, $\mathrm{J} \mathrm{mol}^{-1}$

$\Delta s \quad$ specific entropy change of reaction, $\mathrm{J} \mathrm{mol}^{-1} \mathrm{~K}^{-1}$

$\Delta v \quad$ specific volume change, $\mathrm{m}^{3} \mathrm{~mol}^{-1}$

$\zeta_{V} \quad$ fractional degree of vaporization, Dimensionless 


\section{Supercripts}

$\begin{array}{ll}r x n & \text { reaction } \\ \sigma & \text { saturation conditions } \\ \theta & \text { reference conditions } \\ \text { vap } & \text { vaporization } \\ * & \text { dimensionless parameter }\end{array}$

\section{Subscripts}

$\begin{array}{ll}A & \text { reactant } \\ B & \text { product } \\ \text { eq } & \text { equilibrium conditions } \\ i & \text { generic component }(i=\mathrm{A}, \mathrm{B}) \\ L & \text { liquid phase } \\ \text { opt } & \text { optimum conditions } \\ \text { tot } & \text { total } \\ V & \text { vapor phase }\end{array}$

\section{Acknowledgements}

The authors would like to acknowledge financial support provided by JNICT through programs CIENCIA (grant BD/2081/92 - IF, PhD fellowship) and PRAXIS XXI (grant $\mathrm{BD} / 5568 / 95, \mathrm{PhD}$ fellowship; and project 2/2.1/BIO/34/94 - Extractive Biocatalysis, research grant).

\section{References}

Bart, H. J., Marr, R., Bauer, U., \& Reisinger, H. (1992). Reactiveextraction of $L$-phenylalanine from an enzymatic solution. In $\mathrm{T}$. Sekine, Solvent Extraction (pp. 1797-1802). Amsterdam: Elsevier.

Cabral, J. M. S. (1991). Extractive removal of product by biocatalysis. In B. Mattiasson, \& O. Holst, Extractive Bioconversions (pp. 207-235). New York: Marcel Dekker.

Davies, B., \& Jeffrey, G. V. (1973). The continuous trans-esterification of ethyl alcohol and butyl acetate in a sieve plate column. Part III. Trans-esterification in a six plate sieve plate column. Transactions of the Institute of Chemical Engineers, 51, 275-280.

De Garmo, J. L., Parulekar, V. N., \& Pinjala, V. (1992). Consider reactive distillation. Chemical Engineering Progress, 43, 43-50.

Groot, W. J., Kraayenbrink, M. R., Waldram, R. H., van der Lans, R. G. J. M., \& Luyben, K. C. A. M. (1992). Ethanol production in an integrated process of fermentation and ethanol recovery by pervaporation. Bioprocess Engineering, 8, 9-111.

Hills, G. A., Macrae, A. R., \& Poulina, R. R. (1990). Ester preparation. Eur. patent no. 0383405 .

Jansen, M. L. (1996). Integration of ion exchange chromatography with an enzymatic reaction. Ph.D. thesis, Technical University of Delft, Netherlands.

Jansen, M. L., van Zessen, E., Straathof, A. J. J., van der Wielen, L. A. M., Luyben, K. C. A. M., \& van der Tweel, W. J. J. (1996). Immobilisation of aminoacylase on an anion exchange column to be used as a chromatographic reactor. Annals of the New York Academy of Sciences, 799, 533-540.

Kemp, R. A., \& Macrae, A. R., (1992). Esterification process. Eur. patent no. 0506159

Laane, C., Tramper, J., \& Lilly, M. D. (Eds.) (1987). Biocatalysis in Organic Media. Amsterdam: Elsevier.
Martinek, K., Klyachko, N. L., Kabanov, A. V., Khmelnitsky, Y. L., \& Levashov, A. V. (1989). Micellar enzymology: its relation to membranology. Biochimica et Biophysica Acta, 981, 161-172.

Marty, A., Chulalaksananukul, W., Willemot, R. M., \& Condoret, J. S. (1992). Kinetics of lipase-catalyzed esterification in supercritical $\mathrm{CO}_{2}$. Biotechnology and Bioengineering, 39, 273-280.

Marty, A., Combes, D., \& Condoret, J. S. (1994). Continuous reactionseparation processes for enzymatic esterification in supercritical carbon dioxide. Biotechnology and Bioengineering, 43, 497-504.

Matsumura, M. (1991). Perstraction. In B. Mattiasson, \& O. Holst, Extractive Bioconversions (pp. 91-131). New York: Marcel Dekker.

Mazzotti, M., Kruglov, A., Neri, B., Gelosa, D., \& Morbidelli, M. (1996). A continuous chromatographic reactor: SMBR. Chemical Engineering Science, 51, 1827-1836.

Paiva, A. L., \& Malcata, F. X. (1994). Process integration involving lipase-catalyzed ester synthesis reactions. Biotechnology Techniques, $8,629-634$.

Paiva, A. L., \& Malcata, F. X. (1996). Integration of reaction and separation with lipases: Biocatalytic Distillation. In F. X. Malcata, Engineering of/with Lipases (pp. 597-611). Dordrecht: Kluwer Academic Publishers.

Paiva, A. L., \& Malcata, F. X. (1997a). Does thermodynamics improve processing when chemical reaction is integrated with physical separation in binary ideal mixtures?. Chemical Engineering Science, 52, 449-452.

Paiva, A. L., \& Malcata, F. X. (1997b). Reversible reaction and diffusion within a porous catalyst slab. Chemical Engineering Science, 52, 4429-4432.

Paiva, A. L., \& Malcata, F. X. (1997c). Integration of reaction and separation with lipases: an overview. Journal of Molecular Catalysis B: Enzymatic, 3, 99-109.

Paiva, A. L., \& Malcata, F. X. (1999). How performance of integrated systems of reaction and separation relates to that of parallel and sequential configurations. Bioprocess Engineering, in press.

Paiva, A. L., van Rossum, D., \& Malcata, F. X. (1998). Cascading reactor-separator sets reduces total time for low yield MichaelisMenten reactions: Model predictions. Biocatalysis and Biotransformations, 16, 205-224.

Paiva, A. L., van Rossum, D., \& Malcata, F. X. (1999). Integrated vs. sequential reaction and separation: contributions for a global analysis. Chemical Engineering Science, in press.

Parvaresh, F., Robert, H., Thomas, D., \& Legoy, M. D. (1992). Gasphase transesterification reactions catalyzed by lipolytic enzymes. Biotechnology and Bioengineering, 39, 467-473.

Perry, R. H., Green, D. W., \& Maloney, J. O. (Eds.) (1984). Perry's Chemical Engineers Handbook. Singapore: McGraw Hill.

Roychoudhury, P. K., Srivastava, A., \& Sahai, V. (1995). Extractive bioconversion of lactic acid. In A. Fiechter, Advances in Biochemical Engineering/Biotechnology (Vol. 53, pp. 61-87) Berlin: Springer.

Smith, J. M., \& van Ness, H. C. (1987). Introduction to Chemical Engineering Thermodynamics. New York: McGraw-Hill.

Strathmann, H., \& Gudernatsch, W. (1991). Continuous removal of ethanol from bioreactor by pervaporation. In B. Mattiasson, \& O. Holst, Extractive Bioconversions (pp. 67-89). New York: Marcel Dekker.

Sundquist, J., Blanch, H. W., \& Wilke, C. R. (1991). Vacuum fermentation. In B. Mattiasson, \& O. Holst, Extractive Bioconversions (pp 237-258). New York: Marcel Dekker.

Takeuchi, K., \& Uraguchi, Y. (1977). Experimental studies of a chromatographic moving bed reactor. Catalytic oxidation of carbon monoxide on activated alumina as a model reaction. Journal of Chemical Engineering of Japan, 10, 455-460.

Tramper, J., Vermue, M. H., Beeftink, H. H., \& von Stockar, U. (1992). Biocatalysis in Non-conventional Media. Amsterdam: Elsevier.

van der Padt, A., Sewalt, J. J. W., \& van't Riet, K. (1996). Membrane bioreactor design to force equilibrium towards a favourable product 
yield. In F. X. Malcata, Engineering of/with Lipases (pp. 130-138). Dordrecht: Kluwer Academic Publishers.

van der Wielen, L. A. M., Straathof, A. J. J., \& Luyben, K. C. A. M. (1993). Adsorptive and chromatographic bioreactors. In M. P. C. Weijnen, \& A. A. H. Drinkenburg, Precision Process Technology (pp. 353-379). Dordrecht: Kluwer Academic Publishers. van der Wielen, L. A. M., Diepen, P. J., Houwers, J., \& Luyben, K. C. A. M. (1996). A countercurrent adsorptive reactor for acidifying bioconversion. Chemical Engineering Science, 51, 2315-2325.

Xu, Z. P., \& Chuang, K. T. (1996). Kinetics of acetic acid esterification over ion exchange catalysis. Canadian Journal of Chemical Engineering, $74,493-500$. 